\title{
Solving Differential Equations of Second Order using Quadratic Legendre Multi-wavelets (QLMW) with Operational Matrix of Integration
}

\author{
Meenu Devi \\ Department of Mathematics \\ and Statistics, Faculty of Science, \\ Gurukula Kangri University \\ Haridwar (UK), India.
}

\author{
S. R. Verma* \\ Department of Mathematics \\ and Statistics, Faculty of Science, \\ Gurukula Kangri University \\ Haridwar (UK), India. \\ ${ }^{*}$ Corresponding Author:
}

\author{
M. P. Singh \\ Department of Mathematics \\ and Statistics, Faculty of Science, \\ Gurukula Kangri University \\ Haridwar (UK), India.
}

\begin{abstract}
In this paper is suggested an efficient method to solve differential equations. Using quadratic Legendre multi-wavelets approximation method, differential equations are converted into the system of algebraic equations with the help of operational matrix of integration and its product. Some illustrative examples are included to show the efficiency and applicability of the method.
\end{abstract}

\section{AMS Subject Classifications: 65T60; 65L10; $65 \mathrm{M} 70$.}

\section{Keywords}

Quadratic Legendre wavelets; Quadratic Legendre multiwavelets; Operational matrix of integration; Differential equations.

\section{INTRODUCTION}

Wavelet is compactly supported [1] square integrable function in time domain and frequency domain. Firstly, wavelet was introduced by N. Ricker [2] in Seismology to provide a time dimension to seismic analysis. Also, it was repeated by E. A. Robinson [3, 4]. But finally wavelet was defined by J. Morlet et al [5, 6]. After that A. Grossman [7], a French theoretical physicist recognized the importance of wavelet transform and developed an inversion formula with the help of admissibility condition. Wavelet is new developing area which is being applied mathematics, medical science, technology and more widely in signal analysis. There is a reason of more success of wavelet and their transform to the comparison of Fourier analysis because Fourier analysis does not contain the local information of non-stationary signals but wavelets examine signals simultaneously in both time and frequency. In recent years, wavelet techniques are used to find out the numerical solution of linear and non-linear problems [10,13]. Current applications of wavelet contain climate analysis, financial denoising, denoising of astronomical images, fast solution of differential equations, computer graphic and so on.

The basic approach initiates from the integral of basis vector $\chi(t)$ which is defined by

$$
\int_{0}^{x} \chi(t) d t \approx P \chi(x)
$$

where, $\quad \chi(t)=\left[\varphi_{1}, \varphi_{2}, \ldots, \varphi_{n}\right]^{T} \quad$ in which the elements $\varphi_{i}(t) ; i=1,2,3, \ldots, n$ are orthonormal basis function on certain interval $[0,1]$, and the matrix $P$ can be uniquely illustrated based on the particular orthonormal function.

In section 2, quadratic Legendre multi-wavelet (QLMW) is introduced. Approximation of any function within interval [0, 1], using QLMW, followed by error analysis, is included in section 3. In section 4, the operational matrix of integration for QLMW is calculated. The product of operational matrix is produced in section 5 . In section 6 , these computations are used to derive a method for solving initial value problem. Finally, the advantages of present method with the help of two examples are shown in section 7 .

\section{THE QUADRATIC LEGENDRE MULTI- WAVELET (QLMW)}

Let $\psi$ be a function of $L_{2}(\$)$, called mother wavelet, satisfying the following properties $[7,8]$ :

$$
\int_{0} \psi(t) d t=0 \text { and } \int_{0}|\psi(t)|^{2} d t=1 .
$$

This is also called unit energy function.

A family of such type of functions constructed from translation and dilation of a single function $\psi$, wavelets $[7,8]$ can be defined as:

$$
\psi_{a, b}(t)=\frac{1}{\sqrt{|a|}} \psi\left(\frac{t-b}{a}\right), \quad a, b \in a \neq 0,
$$

where $a$ and $b$ are dilation and translation parameters respectively.

By discretization $[1,7]$ of these parameters $a=2^{-k}$ and $b=n 2^{-k}$, one gets

$$
\psi_{k, n}(t)=2^{k / 2} \psi\left(2^{k} t-n\right), \quad k, n \in \mathbf{C}
$$


Let $\phi$ be a function of $L_{2}\left({ }^{2}\right)$ space. It is said to be scaling function for $V_{0}$, if it satisfies the following condition

$$
\int_{-\infty}^{\infty} \phi(t) d t=1 \text { and } V_{0}=\underset{k}{\operatorname{span}}\{\phi(t-k)\} .
$$

2.1. Definition: The nested sequence $\left\{V_{j}\right\}_{j=-\infty}^{\infty}$ of the subspaces of $L_{2}($ ) with scaling function $\phi$ is called multiresolution analysis (MRA) if it satisfies the following conditions:

(i) $\lim _{j \rightarrow+\infty} V_{j}=L_{2}(\infty)$ and $\lim _{j \rightarrow-\infty} V_{j}=\{0\}$

(ii) $\phi(t) \in V_{0} \Leftrightarrow \phi\left(2^{j} t\right) \in V_{j}$

(iii) $\{\phi(t-n)\}_{n=-\infty}^{\infty}$ is a Riesz basis of $V_{0}$.

For any orthogonal MRA with a multi-scaling function $\phi$, there exist [12] a multi-wavelet function $\psi$ and a multi-scaling function $\phi$ orthogonal to each other, given as below:

$$
\psi(t)=\sqrt{2} \sum_{n=-\infty}^{n=\infty} b_{n} \phi(2 t-n)
$$

and $\left\{\psi_{j, n}\right\}_{j, n=-\infty}^{\infty}$ forms an orthonormal basis for $L_{2}(\infty)$ under certain condition [11].

For constructing the quadratic Legendre multi-wavelet, when the single scaling function $\phi(t)$ is replaced with vector of scaling function $\Phi(t)$ yields:

$$
\Phi(t)=\left[\phi_{0}(t), \phi_{1}(t), \phi_{2}(t)\right]^{T},
$$

where

$$
\begin{aligned}
& \phi_{0}(t)=1, \quad \phi_{1}(t)=\sqrt{3}(2 t-1) \quad \text { and } \\
& \phi_{2}(t)=\sqrt{5}\left(6 t^{2}-6 t+1\right), \quad 0 \leq t \leq 1 .
\end{aligned}
$$

Since $\Psi(t)=\left[\psi_{0}(t), \psi_{l}(t), \psi_{2}(t)\right]^{T}$ are the corresponding mother wavelet functions [11], using equation (2) one gets the quadratic Legendre wavelet (QLW) as given

below:

$$
\begin{aligned}
& \psi_{0}(t)= \begin{cases}-\frac{1}{3}\left(120 t^{2}-72 t+7\right), & 0 \leq t \leq \frac{1}{2} \\
\frac{1}{3}\left(120 t^{2}-168 t+55\right), & \frac{1}{2} \leq t \leq 1,\end{cases} \\
& \psi_{1}(t)= \begin{cases}\sqrt{3}\left(30 t^{2}-14 t+1\right), & 0 \leq t \leq \frac{1}{2} \\
\sqrt{3}\left(30 t^{2}-46 t+17\right), & \frac{1}{2} \leq t \leq 1,\end{cases}
\end{aligned}
$$

$$
\psi_{2}(t)= \begin{cases}\frac{-\sqrt{5}}{3}\left(48 t^{2}-18 t+1\right), & 0 \leq t \leq \frac{1}{2} \\ \frac{\sqrt{5}}{3}\left(48 t^{2}-78 t+31\right), & \frac{1}{2} \leq t \leq 1,\end{cases}
$$

Translating and dilating the quadratic Legendre wavelet $\Psi(t)$, we get

$$
\psi_{k, n}^{j}(t)=2^{k / 2} \psi^{j}\left(2^{k} t-n\right), \quad k, n, j \in \mathbf{C} \text {. }
$$

The family $\left\{\psi_{k, n}^{j}(t)\right\}$ forms an orthonormal basis for $L_{2}(\infty)$ and subfamily is orthonormal in $L_{2}[0,1]$ for $n=0,1,2, \ldots, 2^{k}-1 ; k=0,1,2, \ldots$ and $j=0,1,2$. If $n=0,1$; $k=0,1$ and $j=0,1,2$; the scaling functions and subfamily members are plotted in the figures 1-12. Here, variable $t$ and family members $\left\{\phi_{0}, \phi_{1}, \phi_{2}, \psi_{00}^{0}, \psi_{00}^{1}, \psi_{00}^{2}, \psi_{10}^{0}, \psi_{10}^{1}, \psi_{10}^{2}, \psi_{11}^{0}, \psi_{11}^{1}, \psi_{11}^{2}\right\}$ are shown along horizontal and vertical axes respectively.

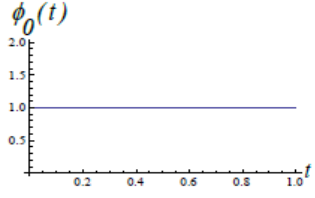

Fig.1

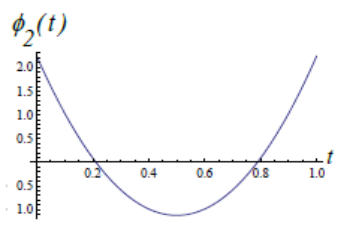

Fig. 3

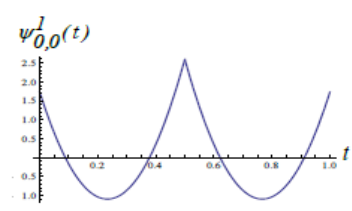

Fig. 5

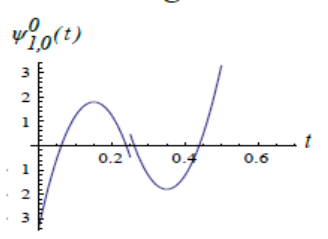

Fig.7

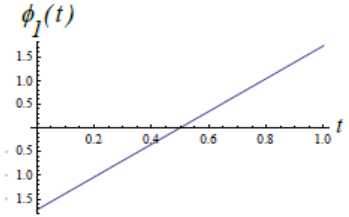

Fig. 2

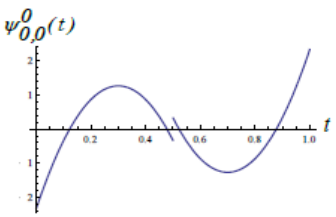

Fig. 4

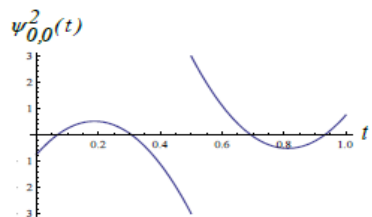

Fig .6

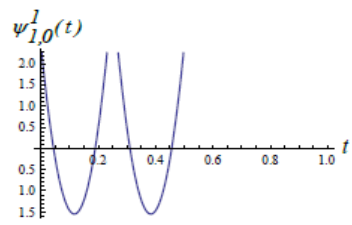

Fig. $8 \psi$ 


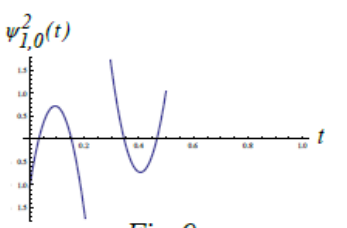

Fig.9

Fig.11

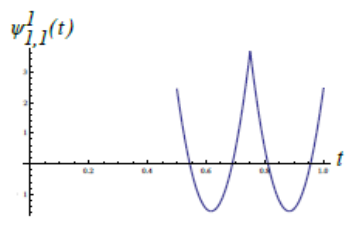

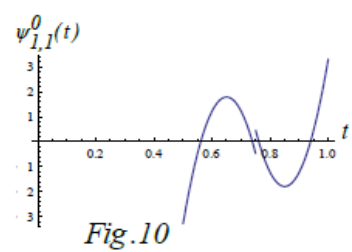

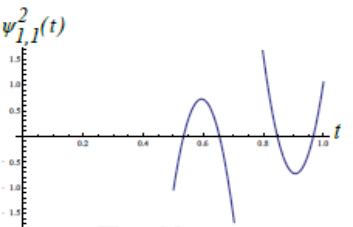

Fig. 12

\section{FUNCTION APPROXIMATIONS}

The function $f(x)$, using quadratic Legendre multi-wavelets in the interval $[0,1]$, is approximated as given below:

$$
f(x)=\sum_{i=0}^{2} c_{i} \phi_{i}+\sum_{k=0}^{\infty} \sum_{j=0}^{2} \sum_{n=0}^{2^{k}-1} c_{k, n}^{j} \psi_{k, n}^{j}(x)
$$

where,

$c_{i}=<f(x), \phi_{i}(x)>$ and $c_{k, n}^{j}=<g(x), \psi_{k, n}^{j}(x)>$. After truncating the infinite series, equation (9) takes the following form:

$$
f(x) \approx \sum_{i=0}^{2} c_{i} \phi_{i}+\sum_{k=0}^{M} \sum_{j=0}^{2} \sum_{n=0}^{2^{k}-1} c_{k, n}^{j} \psi_{k, n}^{j}(x)=C^{T} \Theta(x),
$$

where,

$$
\begin{aligned}
C=\left[c_{0}, c_{1,}, c_{2}, c_{00}^{0}, \ldots, c_{M\left(2^{M}-1\right)}^{0}, c_{00}^{1}, \ldots,\right. & \\
\ldots . & \left.c_{M\left(2^{M}-1\right)}^{1}, c_{00}^{2}, \ldots, c_{M\left(2^{M}-1\right)}^{2}\right]^{T}
\end{aligned}
$$

and

$$
\begin{aligned}
\Theta(x)= & {\left[\phi_{O}, \phi_{1}, \phi_{2}, \psi_{O O}^{o}, \ldots, \psi_{M\left(2^{M-1}\right)}^{o},\right.} \\
& \left.\psi_{O O}^{1}, \ldots, \psi_{M\left(2^{M-1}\right.}^{1}, \psi_{O O}^{2}, \ldots, \psi_{M\left(2^{M-1}\right)}^{2}\right]^{T} .
\end{aligned}
$$

Theorem 3.1 Let the function $f:[0,1] \rightarrow$ and $f \in C^{3}[0,1]$. Then $C^{T} \Theta$ approximates $f$ with mean error bound as follows:

$$
\left\|f-C^{T} \Theta\right\| \leq \frac{1}{3 ! 2^{3 k}} \sup _{x \in[0,1]}\left|f^{\prime \prime \prime}(x)\right|
$$

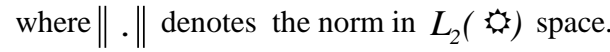

Proof. The proof of this theorem can be seen in [2].

It can be seen from this theorem; the factor $\frac{1}{3 ! 2^{3 k}}$ shows best approximation of function because if $k$ increases then error decreases rapidly.

\section{OPERATIONAL MATRIX OF INTEGRATION OF QLMW}

With the help of equation (12), equation (1) takes the form:

$$
\int_{0}^{x} \Theta(t) d t \approx P_{12 \times 12} \Theta(x)
$$

where, $P_{12 \times 12}$ an operational matrix of integration of QLMW [11] is given below:

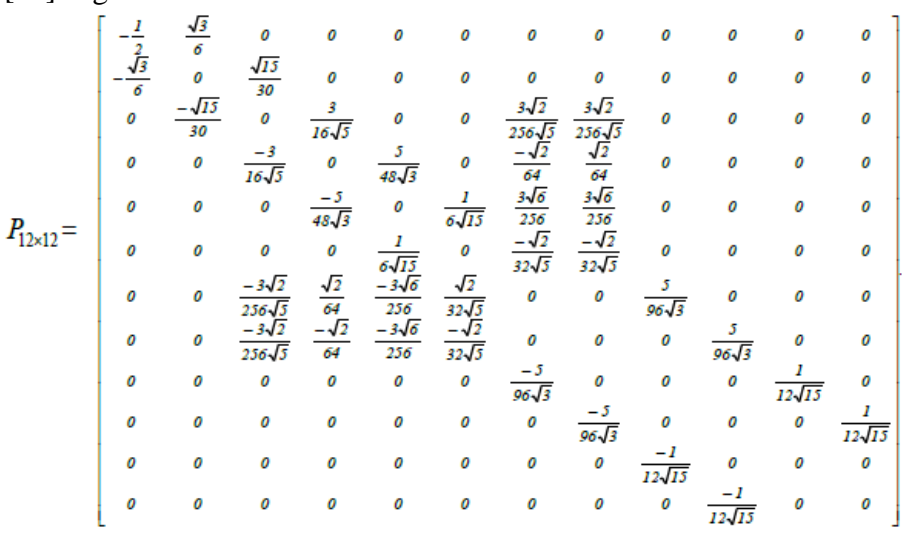

In general

$$
P=\left[\begin{array}{cc}
A_{\left(3.2^{n}\right) \times\left(3.2^{n}\right)} & B_{\left(3.2^{n}\right) \times\left(3.2^{n}\right)} \\
-B_{\left(3.2^{n}\right) \times\left(3.2^{n}\right)} & C_{\left(3.2^{n}\right) \times\left(3.2^{n}\right)}
\end{array}\right],
$$

where order of $P$, given by [11], is $\left(3.2^{n+1}\right) \times\left(3.2^{n+1}\right)$.

\section{THE PRODUCT OF QLMW BASIS VECTOR AND IT'S TRANSPOSE}

In this section the product of operational integration matrix of QLMW is obtained, which is useful in solving non homogeneous differential and integral equations. From equation (12) we have

$$
\Theta(x) \Theta^{T}(x)=\sum_{j=0}^{2} \phi_{j} I_{j}+\sum_{j=0}^{2} \sum_{i=0}^{M} \sum_{k=0}^{2^{M}-1} \psi_{i, k}^{j} I_{i, k}^{j},
$$

where,

$$
\begin{aligned}
& I_{j}=\left[\begin{array}{cc}
A_{j} & B_{j} \\
{\left[B_{j}\right]^{T}} & C_{j}
\end{array}\right]_{\left(3.2^{n+1}\right) \times\left(3.2^{n+1}\right)}, \\
& I_{i, k}^{j}=\left[\begin{array}{cc}
A_{i, k}^{j} & B_{i, k}^{j} \\
{\left[B_{i, k}^{j}\right]^{T}} & C_{i, k}^{j}
\end{array}\right]_{\left(3.2^{n+1}\right) \times\left(3.2^{n+1}\right)}, \text {, } \\
& A_{j}=\left[\begin{array}{cc}
\left(a^{\prime}\right)_{j} & \left(a^{\prime \prime}\right)_{j} \\
{\left[\left(a^{\prime \prime}\right)_{j}\right]^{T}} & \left(a^{\prime \prime \prime}\right)_{j}
\end{array}\right]_{\left(3.2^{n}\right) \times\left(3.2^{n}\right)}, \\
& A_{i . k}^{j}=\left[\begin{array}{cc}
\left(a^{\prime}\right)_{i, k}^{j} & \left(a^{\prime \prime}\right)_{i, k}^{j} \\
{\left[\left(a^{\prime \prime}\right)_{i, k}^{j}\right]^{T}} & \left(a^{\prime \prime \prime}\right)_{i, k}^{j}
\end{array}\right]_{\left(3.2^{n}\right) \times\left(3.2^{n}\right)}, \text {, } \\
& C_{j}=\left[\begin{array}{cc}
\left(c^{\prime}\right)_{j} & \left(c^{\prime \prime}\right)_{j} \\
{\left[\left(c^{\prime \prime}\right)_{j}\right]^{T}} & \left(c^{\prime \prime \prime}\right)_{j}
\end{array}\right]_{\left(3.2^{n}\right) \times\left(3.2^{n}\right)}, \text {, } \\
& C_{i . k}^{j}=\left[\begin{array}{cc}
\left(c^{\prime}\right)_{i, k}^{j} & \left(c^{\prime \prime}\right)_{i, k}^{j} \\
{\left[\left(c^{\prime \prime}\right)_{i, k}^{j}\right]^{T}} & \left(c^{\prime \prime \prime}\right)_{i, k}^{j}
\end{array}\right]_{\left(3.2^{n}\right) \times\left(3.2^{n}\right)} .
\end{aligned}
$$

The order of the elements of the matrices $A_{j}, B_{j}, C_{j}, A_{i . k}^{j}$, $B_{i . k}^{j}, \quad C_{i . k}^{j} \quad$ is $\quad\left(3.2^{n-1}\right) \times\left(3.2^{n-1}\right) . \quad$ For 
$i=0,1 ; j=0,1,2 ; k=0,1$ and $M=1$, the matrices $I_{j}$ and $I_{i, k}^{j}$ are of order $12 \times 12$. Also, the matrices $I_{0}, A_{0}$ are identities and $A_{1,1}^{1}, A_{l, 1}^{2}$ are null matrices. Thus, the entries of matrices $A_{j}, A_{i, k}^{j}$ are given below:

$$
\begin{aligned}
& \left(a^{\prime}\right)_{l}=\left[\begin{array}{ccc}
0 & 1 & 0 \\
1 & 0 & \frac{2}{\sqrt{5}} \\
0 & \frac{2}{\sqrt{5}} & 0
\end{array}\right], \quad\left(a^{\prime \prime}\right)_{l}=\left[\begin{array}{ccc}
0 & 0 & 0 \\
0 & 0 & 0 \\
\frac{9 \sqrt{3}}{8 \sqrt{5}} & 0 & 0
\end{array}\right], \\
& \left(a^{\prime \prime \prime}\right)_{l}=\left[\begin{array}{ccc}
0 & \frac{17}{24} & 0 \\
\frac{17}{24} & 0 & \frac{2}{3 \sqrt{5}} \\
0 & \frac{2}{3 \sqrt{5}} & 0
\end{array}\right], \quad\left(a^{\prime}\right)_{2}=\left[\begin{array}{ccc}
0 & 0 & 1 \\
0 & \frac{2}{\sqrt{5}} & 0 \\
1 & 0 & \frac{2 \sqrt{5}}{7}
\end{array}\right] \text {, } \\
& \left(a^{\prime \prime}\right)_{2}=\left[\begin{array}{ccc}
0 & 0 & 0 \\
\frac{9 \sqrt{3}}{8 \sqrt{5}} & 0 & 0 \\
0 & \frac{3 \sqrt{3}}{7} & 0
\end{array}\right], \quad\left(a^{\prime \prime \prime}\right)_{2}=\left[\begin{array}{ccc}
\frac{58}{63 \sqrt{5}} & 0 & \frac{173}{504} \\
0 & -\frac{4}{7 \sqrt{5}} & 0 \\
\frac{173}{504} & 0 & \frac{-26 \sqrt{5}}{63}
\end{array}\right] \text {, } \\
& \left(a^{\prime}\right)_{0,0}^{o}=\left[\begin{array}{ccc}
0 & 0 & 0 \\
0 & 0 & \frac{9 \sqrt{3}}{8 \sqrt{5}} \\
0 & \frac{9 \sqrt{3}}{8 \sqrt{5}} & 0
\end{array}\right], \quad\left(a^{\prime \prime}\right)_{0,0}^{o}=\left[\begin{array}{ccc}
1 & 0 & 0 \\
0 & \frac{17}{24} & 0 \\
\frac{58}{63 \sqrt{5}} & 0 & \frac{173}{504}
\end{array}\right], \\
& \left(a^{\prime \prime \prime}\right)_{0,0}^{o}=\left[\begin{array}{ccc}
0 & \frac{2}{21 \sqrt{3}} & 0 \\
\frac{2}{21 \sqrt{3}} & 0 & \frac{-31}{168 \sqrt{15}} \\
0 & \frac{-31}{168 \sqrt{15}} & 0
\end{array}\right],\left(a^{\prime}\right)_{0,0}^{l}=\left[\begin{array}{ccc}
0 & 0 & 0 \\
0 & 0 & 0 \\
0 & 0 & \frac{3 \sqrt{3}}{7}
\end{array}\right], \\
& \left(a^{\prime \prime}\right)_{o, 0}^{\prime}=\left[\begin{array}{ccc}
0 & 1 & 0 \\
\frac{17}{24} & 0 & \frac{2}{3 \sqrt{5}} \\
0 & -\frac{4}{7 \sqrt{5}} & 0
\end{array}\right],\left(a^{\prime \prime \prime}\right)_{0,0}^{\prime}=\left[\begin{array}{ccc}
\frac{2}{21 \sqrt{3}} & 0 & 0 \\
0 & \frac{3}{7 \sqrt{3}} & 0 \\
\frac{-31}{168 \sqrt{15}} & 0 & \frac{52}{21 \sqrt{3}}
\end{array}\right], \\
& \left(a^{\prime}\right)_{0,0}^{2}=\left[\begin{array}{ccc}
0 & 0 & 0 \\
0 & 0 & 0 \\
0 & 0 & 0
\end{array}\right], \quad\left(a^{\prime \prime}\right)_{0,0}^{2}=\left[\begin{array}{ccc}
0 & 0 & 1 \\
0 & \frac{2}{3 \sqrt{5}} & 0 \\
\frac{173}{504} & 0 & \frac{-23 \sqrt{5}}{63}
\end{array}\right] \text {, } \\
& \left(a^{\prime \prime \prime}\right)_{0,0}^{2}=\left[\begin{array}{ccc}
0 & \frac{-31}{168 \sqrt{15}} & \frac{3}{16 \sqrt{10}} \\
\frac{-31}{168 \sqrt{15}} & 0 & \frac{52}{21 \sqrt{3}} \\
0 & \frac{52}{21 \sqrt{3}} & 0
\end{array}\right] \text {. }
\end{aligned}
$$

Remaining twelve matrices are the generalization of the following six matrices $X_{1}, X_{2}, X_{3}, X_{4}, X_{5}, X_{6}$ :

$$
\begin{aligned}
& X_{1}=\left[\begin{array}{ccc}
0 & 0 & 0 \\
0 & 0 & \frac{9 \sqrt{3}}{64 \sqrt{10}} \\
0 & \frac{9 \sqrt{3}}{64 \sqrt{10}} & \frac{(-1)^{1+1} 27}{16 \sqrt{2}}
\end{array}\right], \quad X_{2}=\left[\begin{array}{ccc}
\frac{(-1)^{1+1} 3 \sqrt{3}}{16 \sqrt{2}} & \frac{27}{64 \sqrt{2}} & \frac{(-1)^{1+1} 3 \sqrt{3}}{8 \sqrt{10}} \\
\frac{24}{16 \sqrt{10}} & \frac{(-1)^{1+1} 63 \sqrt{3}}{64 \sqrt{10}} & \frac{27}{64 \sqrt{2}}
\end{array}\right], \\
& X_{3}=\left[\begin{array}{ccc}
\frac{(-1)^{1+1} 3}{4 \sqrt{2}} & \frac{3 \sqrt{3}}{16 \sqrt{2}} & \frac{(-1)^{1} 3}{16 \sqrt{10}} \\
\frac{3 \sqrt{3}}{16 \sqrt{2}} & \frac{(-1)^{1} 27}{64 \sqrt{2}} & \frac{-57 \sqrt{3}}{64 \sqrt{2}} \\
\frac{(-1)^{1} 3}{16 \sqrt{10}} & \frac{-57 \sqrt{3}}{64 \sqrt{2}} & \frac{(-1)^{1} 3}{4 \sqrt{2}}
\end{array}\right], \quad X_{4}=\left[\begin{array}{ccc}
0 & 0 & 0 \\
0 & 0 & 0 \\
0 & 0 & \frac{3 \sqrt{3}}{112 \sqrt{2}}
\end{array}\right], \\
& X_{5}=\left[\begin{array}{ccc}
0 & 0 & 0 \\
0 & 0 \\
\frac{(-1)^{+1} \sqrt{15}}{28 \sqrt{2}} & \frac{9 \sqrt{5}}{112 \sqrt{2}} & \frac{(-1)^{1+1} \sqrt{3}}{14 \sqrt{2}}
\end{array}\right], \quad X_{6}=\left[\begin{array}{ccc}
\frac{5}{7 \sqrt{6}} & (-1)^{1+1} \frac{15}{28 \sqrt{2}} & \frac{\sqrt{10}}{7 \sqrt{3}} \\
(-1)^{1+1} \frac{15}{28 \sqrt{2}} & \frac{45 \sqrt{3}}{112 \sqrt{2}} & \frac{-3 \sqrt{5}}{14 \sqrt{2}} \\
\frac{\sqrt{10}}{7 \sqrt{3}} & \frac{-3 \sqrt{5}}{14 \sqrt{2}} & \frac{2 \sqrt{2}}{7 \sqrt{3}}
\end{array}\right] .
\end{aligned}
$$

such that

$$
\begin{aligned}
& X_{I}=\left\{\begin{array}{ll}
\left(a^{\prime}\right)_{1,0}^{0}, & l=0 \\
\left(a^{\prime}\right)_{1,0}^{l}, & l=1
\end{array},\right. \\
& X_{2}=\left\{\begin{array}{ll}
\left(a^{\prime \prime \prime}\right)_{1,0}, & l=0 \\
\left(a^{\prime \prime}\right)_{1,0}^{\prime} & l=1
\end{array},\right. \\
& X_{3}=\left\{\begin{array}{l}
\left(a^{\prime \prime \prime \prime} h_{, 0}, l=0\right. \\
\left(a^{\prime \prime \prime}\right)_{1,0}^{\prime}, l=1
\end{array},\right. \\
& X_{4}=\left\{\begin{array}{ll}
\left(a^{\prime}\right)_{l, o}^{2}, & l=0 \\
\left(a^{\prime}\right)_{l, l}^{o}, l=1 & ,
\end{array},\right. \\
& X_{5}=\left\{\begin{array}{ll}
\left(a^{\prime \prime}\right)_{1,0}^{2}, & l=0 \\
\left(a^{\prime \prime}\right)_{1,1}^{p}, & l=1
\end{array},\right. \\
& X_{6}=\left\{\begin{array}{l}
\left(a^{\prime \prime \prime} h_{h, o}, l=0\right. \\
\left(a^{\prime \prime \prime} \rho_{h, l}, l=1\right.
\end{array},\right.
\end{aligned}
$$

Thus, the entries of $C_{j}$ and $C_{i, k}^{j}$ are achieved. Also, it can be seen that $C_{0}$ is obtained as identity matrix, $\left(c^{\prime}\right)_{1,0}^{l}$, $\left(c^{\prime \prime \prime}\right)_{1,1}^{l}, \quad\left(c^{\prime \prime \prime}\right)_{1,0}^{0}, \quad\left(c^{\prime}\right)_{1,1}^{2}$ as null matrices and the following as non-zero matrices:

$$
\begin{aligned}
& \left(c^{\prime}\right)_{1}=\left[\begin{array}{ccc}
\frac{-\sqrt{3}}{2} & 0 & \frac{17}{48} \\
0 & \frac{\sqrt{3}}{2} & 0 \\
\frac{17}{48} & 0 & \frac{-\sqrt{3}}{2}
\end{array}\right], \quad\left(c^{\prime \prime}\right)_{1}=\left[\begin{array}{ccc}
0 & 0 & 0 \\
\frac{17}{48} & 0 & 0 \\
0 & \frac{1}{3 \sqrt{5}} & 0
\end{array}\right], \\
& \left(c^{\prime \prime \prime}\right)_{1}=\left[\begin{array}{ccc}
\frac{\sqrt{3}}{2} & 0 & \frac{1}{3 \sqrt{5}} \\
0 & \frac{-\sqrt{3}}{2} & 0 \\
\frac{1}{3 \sqrt{5}} & 0 & \frac{\sqrt{3}}{2}
\end{array}\right], \quad\left(c^{\prime}\right)_{1,0}^{o}=\left[\begin{array}{ccc}
0 & 0 & \frac{2 \sqrt{2}}{21 \sqrt{3}} \\
0 & 0 & 0 \\
\frac{2 \sqrt{2}}{21 \sqrt{3}} & 0 & 0
\end{array}\right], \\
& \left(c^{\prime \prime}\right)_{1,0}^{0}=\left[\begin{array}{ccc}
0 & 0 & 0 \\
0 & 0 & 0 \\
0 & \frac{-31}{84 \sqrt{30}} & 0
\end{array}\right], \quad\left(c^{\prime \prime}\right)_{1,0}^{1}=\left[\begin{array}{ccc}
0 & 0 \\
\frac{2 \sqrt{3}}{21 \sqrt{2}} & 0 & 0 \\
0 & 0 & 0
\end{array}\right], \\
& \left(c^{\prime}\right)_{1,0}^{2}=\left[\begin{array}{ccc}
\frac{2 \sqrt{2}}{21 \sqrt{3}} & 0 & 0 \\
0 & 0 & 0 \\
0 & 0 & \frac{3 \sqrt{6}}{7}
\end{array}\right], \quad\left(c^{\prime}\right)_{1,1}^{0}=\left[\begin{array}{ccc}
0 & 0 & 0 \\
0 & \frac{2 \sqrt{2}}{21 \sqrt{3}} & 0 \\
0 & 0 & 0
\end{array}\right],
\end{aligned}
$$




$$
\begin{aligned}
& \left(c^{\prime \prime}\right)_{1,1}^{0}=\left[\begin{array}{ccc}
0 & 0 & 0 \\
0 & 0 & \frac{-31}{84 \sqrt{30}} \\
0 & 0 & 0
\end{array}\right], \quad\left(c^{\prime \prime \prime}\right)_{1,1}^{0}=\left[\begin{array}{ccc}
\frac{3 \sqrt{6}}{7} & 0 & 0 \\
0 & 0 & 0 \\
0 & 0 & \frac{52 \sqrt{2}}{21 \sqrt{3}}
\end{array}\right], \\
& \left(c^{\prime \prime \prime}\right)_{1,1}^{2}=\left[\begin{array}{ccc}
0 & 0 & \frac{52 \sqrt{2}}{21 \sqrt{3}} \\
0 & 0 & 0 \\
\frac{52 \sqrt{2}}{21 \sqrt{3}} & 0 & 0
\end{array}\right], \quad\left(c^{\prime \prime}\right)_{1,0}^{2}=\left[\begin{array}{ccc}
0 & \frac{-31}{84 \sqrt{30}} & 0 \\
0 & 0 & 0 \\
0 & 0 & 0
\end{array}\right]=\left[\left(c^{\prime \prime}\right)_{1,1}^{2}\right]^{T}, \\
& \left(c^{\prime \prime}\right)_{1,1}^{l}=\left[\begin{array}{ccc}
0 & 0 & 0 \\
0 & \frac{52 \sqrt{2}}{21 \sqrt{3}} & 0 \\
0 & 0 & 0
\end{array}\right]=\left(c^{\prime \prime \prime}\right)_{1,0}^{2},\left(c^{\prime}\right)_{1,1}^{l}=\left[\begin{array}{ccc}
0 & 0 & \frac{-31}{84 \sqrt{30}} \\
0 & 0 & 0 \\
\frac{-31}{84 \sqrt{30}} & 0 & 0
\end{array}\right]=\left(c^{\prime \prime \prime}\right)_{1,0}^{l} \text {. }
\end{aligned}
$$

Similarly, corresponding to the entries of $C_{j}$ and $C_{i, k}^{j}$ the twelve matrices are the generalization of the following six matrices $Z_{1}, Z_{2}, Z_{3}, Z_{4}, Z_{5}, Z_{6}$ :

$$
\begin{aligned}
& \mathrm{Z}_{1}=(\sqrt{15})^{l}\left[\begin{array}{ccc}
\frac{29}{126 \sqrt{5}} & 0 & \frac{17 \sqrt{5}}{32 \sqrt{3}}\left(-\frac{1}{15}\right)^{1} \\
0 & \frac{29}{126 \sqrt{5}} & 0 \\
\frac{17 \sqrt{5}}{32 \sqrt{3}}\left(-\frac{1}{15}\right)^{1} & 0 & \frac{-1}{7 \sqrt{5}}
\end{array}\right], \\
& Z_{2}=(\sqrt{15})^{l}\left[\begin{array}{ccc}
0 & \frac{173}{2016} & 0 \\
\frac{17 \sqrt{5}}{32 \sqrt{3}}\left(\frac{1}{15}\right)^{1} & 0 & \frac{173}{2016}
\end{array}\right] \text {, } \\
& 0 \quad \frac{1}{2 \sqrt{3}}\left(\frac{1}{15}\right)^{1+1} \quad 0 \\
& Z_{3}=(\sqrt{15})^{l}\left[\begin{array}{ccc}
\frac{-1}{7 \sqrt{5}} & 0 & \frac{1}{2 \sqrt{3}}\left(\frac{-1}{15}\right)^{1} \\
0 & \frac{-13}{126 \sqrt{5}}(5)^{1} & 0 \\
\frac{1}{2 \sqrt{3}}\left(\frac{-1}{15}\right)^{1} & 0 & \frac{-13}{126 \sqrt{5}}(5)^{1}
\end{array}\right] \text {, } \\
& Z_{4}=\left(\frac{1}{2 \sqrt{5}}\right)^{l}\left[\begin{array}{ccc}
\frac{5}{189}\left(\frac{-547}{5}\right)^{1} & 0 & \frac{17\left(5^{1}\right)}{24 \sqrt{3}} \\
0 & \frac{5}{189}\left(\frac{-547}{5}\right)^{1} & 0 \\
\frac{17\left(5^{1}\right)}{24 \sqrt{3}} & 0 & \frac{11}{21}\left(\frac{-19}{11}\right)^{1}
\end{array}\right] \text {, } \\
& Z_{5}=\left[\begin{array}{ccc}
0 & \frac{-173 \sqrt{5}}{1512}\left(\frac{2}{\sqrt{5}}\right)^{1} & 0 \\
\frac{17}{24 \sqrt{3}}\left(\frac{-2}{\sqrt{5}}\right)^{-1} & 0 & \frac{-173 \sqrt{5}}{1512}\left(\frac{2}{\sqrt{5}}\right)^{1} \\
0 & \frac{17}{24 \sqrt{3}}\left(\frac{-2}{\sqrt{5}}\right)^{-1} & 0
\end{array}\right],
\end{aligned}
$$

$$
Z_{6}=(\sqrt{5})^{k}\left[\begin{array}{ccc}
\frac{-11}{21}\left(\frac{-19}{110}\right)^{1} & 0 & \frac{2}{3 \sqrt{15}}\left(\frac{-1}{2}\right)^{1} \\
0 & \frac{193}{189}\left(\frac{41}{386}\right)^{1} & 0 \\
\frac{2}{3 \sqrt{15}}\left(\frac{-1}{2}\right)^{1} & 0 & -\frac{193}{189}\left(\frac{41}{386}\right)^{1}
\end{array}\right],
$$

such that

$$
\begin{array}{ll}
Z_{1}=\left\{\begin{array}{l}
\left(c^{\prime}\right)_{2}, l=0 \\
\left(c^{\prime}\right)_{0,0}^{l}, l=1,
\end{array}\right. & Z_{2}=\left\{\begin{array}{l}
\left(c^{\prime \prime}\right)_{2}, l=0 \\
\left(c^{\prime \prime}\right)_{0,0}^{l}, l=l,
\end{array}\right. \\
Z_{3}=\left\{\begin{array}{l}
\left(c^{\prime \prime \prime}\right)_{2}, l=0 \\
\left(c^{\prime \prime \prime}\right)_{0,0}^{l}, l=1
\end{array}\right. & Z_{4}=\left\{\begin{array}{l}
\left(c^{\prime}\right)_{0,0}^{o}, l=0 \\
\left(c^{\prime}\right)_{0,0}^{2}, l=1
\end{array}\right. \\
Z_{5}=\left\{\begin{array}{l}
\left(c^{\prime \prime}\right)_{0,0}^{o}, l=0 \\
\left(c^{\prime \prime}\right)_{0,0}^{2}, l=1
\end{array}\right. & Z_{6}=\left\{\begin{array}{l}
\left(c^{\prime \prime \prime}\right)_{0,0}^{o}, l=0 \\
\left(c^{\prime \prime \prime}\right)_{0,0}^{2}, l=1
\end{array}\right.
\end{array}
$$

And in equation (15) the matrix $B_{0}$ is the null matrix while the other matrices $B_{j}, B_{i . k}^{j} ; i, k=0,1 ; j=0,1,2$ are given below:

$$
B_{1}=\left[\begin{array}{cccccc}
0 & 0 & 0 & 0 & 0 & 0 \\
0 & 0 & 0 & 0 & 0 & 0 \\
\frac{9 \sqrt{3}}{64 \sqrt{10}} & \frac{9 \sqrt{3}}{64 \sqrt{10}} & 0 & 0 & 0 & 0 \\
\frac{-3 \sqrt{3}}{16 \sqrt{2}} & \frac{3 \sqrt{3}}{16 \sqrt{2}} & 0 & 0 & 0 & 0 \\
\frac{27}{64 \sqrt{2}} & \frac{27}{64 \sqrt{2}} & 0 & 0 & 0 & 0 \\
\frac{-3 \sqrt{3}}{8 \sqrt{10}} & \frac{3 \sqrt{3}}{8 \sqrt{10}} & 0 & 0 & 0 & 0
\end{array}\right]
$$$$
Y_{I}=(\sqrt{15})^{l}\left[\begin{array}{cccccc}
0 & 0 & 0 & 0 & 0 & 0 \\
\frac{9 \sqrt{3}}{64 \sqrt{10}} & \frac{9 \sqrt{3}}{64 \sqrt{10}} & 0 & 0 & 0 & 0 \\
-\frac{27}{64 \sqrt{2}}\left(\frac{7}{15}\right)^{1} & \frac{27}{64 \sqrt{2}}\left(\frac{7}{15}\right)^{1} & \frac{3 \sqrt{3}}{112 \sqrt{2}} & \frac{3 \sqrt{3}}{112 \sqrt{2}} & 0 & 0 \\
\frac{27}{16 \sqrt{10}}\left(\frac{1}{9}\right)^{1} & \frac{27}{16 \sqrt{10}}\left(\frac{1}{9}\right)^{1} & \frac{-\sqrt{15}}{28 \sqrt{2}} & \frac{\sqrt{15}}{28 \sqrt{2}} & 0 & 0 \\
-\frac{63 \sqrt{3}}{64 \sqrt{10}}\left(\frac{-1}{7}\right)^{1} & \frac{63 \sqrt{3}}{64 \sqrt{10}}\left(\frac{-1}{7}\right)^{1} & \frac{9 \sqrt{5}}{112 \sqrt{2}} & \frac{9 \sqrt{5}}{112 \sqrt{2}} & 0 & 0 \\
\frac{27}{64 \sqrt{2}}\left(-\frac{19}{45}\right)^{1} & \frac{27}{64 \sqrt{2}}\left(-\frac{19}{45}\right)^{1} & \frac{\sqrt{3}}{14 \sqrt{2}} & \frac{\sqrt{3}}{14 \sqrt{2}} & 0 & 0
\end{array}\right] \text {, }
$$$$
Y_{2}=\left(\frac{2}{\sqrt{5}}\right)^{\prime}\left[\begin{array}{cccccc}
0 & 0 & 0 & 0 & 0 & 0 \\
\frac{-3 \sqrt{3}}{16 \sqrt{2}} & \frac{3 \sqrt{3}}{16 \sqrt{2}} & 0 & 0 & 0 & 0 \\
\frac{27}{16 \sqrt{10}}\left(\frac{5}{8}\right)^{1} & \frac{27}{16 \sqrt{10}}\left(\frac{5}{8}\right)^{1} & \frac{-\sqrt{15}}{28 \sqrt{2}} & \frac{\sqrt{15}}{28 \sqrt{2}} & 0 & 0 \\
\frac{-3}{4 \sqrt{2}}\left(-\frac{1}{8}\right)^{1} & \frac{3}{4 \sqrt{2}}\left(-\frac{1}{8}\right)^{1} & \frac{5}{7 \sqrt{6}}\left(\frac{1}{2}\right)^{1} & \frac{5}{7 \sqrt{6}}\left(\frac{1}{2}\right)^{1} & 0 & 0 \\
\frac{3 \sqrt{3}}{16 \sqrt{2}}\left(\frac{-19}{8}\right)^{1} & \frac{3 \sqrt{3}}{16 \sqrt{2}}\left(\frac{-19}{8}\right)^{1} & \frac{-15}{28 \sqrt{2}}\left(\frac{1}{2}\right)^{1} & \frac{15}{28 \sqrt{2}}\left(\frac{1}{2}\right)^{1} & 0 & 0 \\
\frac{3(10)^{1}}{16 \sqrt{10}} & \frac{-3(10)^{1}}{16 \sqrt{10}} & \frac{\sqrt{10}}{7 \sqrt{3}} & \frac{\sqrt{10}}{7 \sqrt{3}} & 0 & 0
\end{array}\right]
$$ 


$$
\begin{aligned}
& Y_{3}=\left[\begin{array}{cccccc}
0 & 0 & 1 & 0 & 0 & 0 \\
\frac{17}{48} & 0 & \frac{(-1)^{1} \sqrt{3}}{2} & 0 & \frac{1}{3 \sqrt{5}} & 0 \\
\frac{(-1)^{1} 17}{32 \sqrt{3}} & 0 & \frac{-1}{7 \sqrt{5}} & 0 & \frac{(-1)^{1}}{2 \sqrt{3}} & 0 \\
\frac{17}{24 \sqrt{3}} & 0 & \frac{(-1)^{1+1} 11}{21} & 0 & \frac{2}{3 \sqrt{15}} & 0 \\
\frac{(-1)^{1+1} 17}{96} & 0 & \frac{-\sqrt{3}}{7} & 0 & \frac{(-1)^{1+1}}{6 \sqrt{5}} & 0 \\
\frac{(-1)^{1} 17 \sqrt{5}}{48 \sqrt{3}} & 0 & \frac{(-1)^{1} 19}{42 \sqrt{5}} & 0 & \frac{-1}{3 \sqrt{5}} & 0
\end{array}\right], \\
& Y_{4}=\left[\begin{array}{cccccc}
0 & 0 & 0 & 0 & 1 & 0 \\
0 & 0 & \frac{1}{3 \sqrt{5}} & 0 & \frac{(-1)^{1} \sqrt{3}}{2} & 0 \\
\frac{173}{2016} & 0 & \frac{(-1)^{1}}{2 \sqrt{3}} & 0 & \frac{-13 \sqrt{5}}{126} & 0 \\
\frac{(-1)^{1} 173 \sqrt{5}}{1512} & 0 & \frac{2}{3 \sqrt{15}} & 0 & \frac{(-1)^{1+1} 193}{189} & 0 \\
\frac{173 \sqrt{5}}{672 \sqrt{2}} & 0 & \frac{(-1)^{1+1}}{6 \sqrt{5}} & 0 & \frac{-65}{42 \sqrt{3}} & 0 \\
\frac{(-1)^{1} 173}{756} & 0 & \frac{1}{3 \sqrt{3}} & 0 & \frac{(-1)^{1+1} 41 \sqrt{5}}{378} & 0
\end{array}\right] \text {, } \\
& Y_{5}=\left[\begin{array}{cccccc}
\frac{1}{(-1)^{1} \sqrt{3}} & 0 & 0 & 0 & 0 & 0 \\
\frac{29}{26 \sqrt{5}} & 0 & \frac{17}{48} & 0 & 0 & 0 \\
\frac{(-1)^{1+1} 5}{189} & 0 & \frac{(17) \sqrt{3}}{24 \sqrt{3}} & 0 & \frac{(-1)^{1} 173 \sqrt{5}}{1512} & 0 \\
\frac{29}{42 \sqrt{3}} & 0 & \frac{(-1)^{1+1} 17}{96} & 0 & \frac{173 \sqrt{5}}{673 \sqrt{3}} & 0 \\
\frac{(-1)^{1} 547}{378 \sqrt{5}} & 0 & \frac{-17 \sqrt{5}}{48 \sqrt{3}} & 0 & \frac{(-1)^{1} 173}{756} & 0
\end{array}\right]
\end{aligned}
$$

When $l=0$ then $\quad \mathrm{Y}_{3}=B_{1,0}^{2}, \quad \mathrm{Y}_{4}=B_{1,1}^{l} \quad$ and $\quad \mathrm{Y}_{5}=B_{1,0}^{0} \quad$ and if $l=1$ then $\mathrm{Y}_{3}, \mathrm{Y}_{4}, \mathrm{Y}_{5}$ are related to $B_{l, l}^{0}, B_{l, l}^{2}, B_{l, 0}^{l}$ respectively with cyclic operation of columns $c_{s+1}=c_{s}, s=1,2,3,4,5$ and

$Y_{l}=\left\{\begin{array}{ll}B_{2}, & l=0 \\ B_{0,0}^{l}, & l=1\end{array}, \quad Y_{2}=\left\{\begin{array}{ll}B_{0,0}^{0}, & l=0 \\ B_{0,0}^{2}, & l=1\end{array}\right.\right.$.

With the help of equation (11) and the matrices $I_{j}$ and $I_{i, k}^{j}$, the equation (34) of [9] takes the form:

$$
\Theta(x) \Theta^{T}(x) C \approx \tilde{C} \Theta(x) .
$$

\section{METHOD OF SOLUTION OF INITIAL VALUE PROBLEMS}

In this section is suggested an approximate solution of second order differential equation with initial conditions using QLMW and operational matrix of integration.

Consider the equation

$$
a(x) y^{\prime \prime}+b(x) y^{\prime}+c(x) y(x)=f(x)
$$

with $y(0)=\alpha$ and $y^{\prime}(0)=\beta$, where, $a, b, c$ and $f$ are function of $x$ or constants.

First, approximating $a, b, c$ and $f$ by using equation (10) we get

$a(x)=A^{T} \Theta(x), b(x)=B^{T} \Theta(x), c(x)=C^{T} \Theta(x)$,

$f(x)=F^{T} \Theta(x), y^{\prime}(0)=E_{0}^{T} \Theta(x)$ and $y(0)=D_{0}^{T} \Theta(x)$.

Now

$$
y^{\prime \prime}(x)=Y^{T} \Theta(x)
$$

and

$$
\begin{aligned}
y^{\prime}(x) & =\int_{0}^{x} y^{\prime \prime}(x) d x+y^{\prime}(0) \\
& \approx Y^{T} P \Theta(x)+E_{o}{ }^{T} \Theta(x) \\
y(x) & =\int_{0}^{x} y^{\prime}(x) d x+y(0) \\
& \approx Y^{T} P^{2} \Theta(x)+E_{0}{ }^{T} P \Theta(x)+D_{0}^{T} \Theta(x)
\end{aligned}
$$

By using equations (18) and (19), the equation (17) yields $\Theta^{T}(x) F=A^{T} \Theta(x) \Theta^{T}(x) Y+B^{T} \Theta(x)\left(\Theta^{T}(x) P^{T} Y\right.$

$$
\begin{gathered}
\left.+\Theta^{T}(x) E_{0}\right)+C^{T} \Theta(x)\left(\Theta^{T}(x)\left[P^{T}\right]^{2} Y\right. \\
\left.+\Theta^{T}(x) P E_{0}^{T}+\Theta^{T}(x) D_{0}\right) \\
=A^{T} \Theta(x) \Theta^{T}(x) Y+B^{T} \Theta(x) \Theta^{T}(x) P^{T} Y \\
+B^{T} \Theta(x) \Theta^{T}(x) E_{0}+C^{T} \Theta(x) \Theta^{T}(x)\left[P^{T}\right]^{2} Y \\
+C^{T} \Theta(x) \Theta^{T}(x) P E_{0}^{T}+C^{T} \Theta(x) \Theta^{T}(x) D_{0} .
\end{gathered}
$$

Now using the product operation matrix $\tilde{C}$ of equation (16) we obtained

$$
\begin{gathered}
\tilde{A} Y+\widetilde{B} P^{T} Y+\widetilde{B} E_{0}+\tilde{C} P^{2 T} Y+\widetilde{C} P E_{o}^{T}+\tilde{C} D_{o}=F \\
\text { or } \quad \tilde{A} Y+\widetilde{B} P^{T} Y+\widetilde{C} P^{2 T} Y=F-\widetilde{B} E_{0}-\tilde{C} P E_{0}^{T}-\tilde{C} D_{0} .
\end{gathered}
$$

After finding $Y$ the approximated solution of differential equation can be obtained.

\section{EXAMPLES}

The following two examples have been solved by proposed method in this paper.

Example 1.1 Consider

$$
x y^{\prime \prime}+2 y^{\prime}-6 x=0
$$

with $y(0)=0, y^{\prime}(0)=0$.

Putting,

$$
\begin{gathered}
y^{\prime \prime}(x)=Y^{T} \Theta(x) \\
Y=\left[y_{0,} y_{1,} y_{2,} y_{3,} y_{4,} y_{5}, y_{6}, y_{7}, y_{8}, y_{9}, y_{10}, y_{11}\right]^{T}
\end{gathered}
$$

and

$\Theta(x)=\left[\phi_{0}, \phi_{1}, \phi_{2}, \psi_{00}^{0}, \psi_{00}^{1}, \psi_{00}^{2}, \psi_{10}^{0}, \psi_{10}^{1}, \psi_{10}^{2}, \psi_{11}^{0}, \psi_{11}^{1}, \psi_{11}^{2}\right]^{T}$.

Using initial condition, we can get

$$
y^{\prime}(x)=Y^{T} P \Theta(x), y(x)=Y^{T} P^{2} \Theta(x)
$$


and

$$
x=E^{T} \Theta(x), \quad 2=F^{T} \Theta(x), \quad 6=G^{T} \Theta(x) .
$$

Now inserting equations (22)-(26) into equation (21) we obtain

$$
\Theta^{T}\left(\tilde{E}+\tilde{F} P^{T}\right) Y=\Theta^{T} \tilde{G} E .
$$

Which is hold for each $X$ in defined interval, therefore we get

$$
\left(\widetilde{E}+\widetilde{F} P^{T}\right) Y=\widetilde{G} E,
$$

so yields

$$
Y=[2,0,0,0,0,0,0,0,0,0,0,0]^{\mathrm{T}} .
$$

By virtue of equation (25) we get exact solution

$$
y(x)=x^{2} \text {. }
$$

Example 1.2 Consider

$$
\begin{aligned}
& \qquad y^{\prime \prime}(x)=1, \\
& \text { with } y(0)=0, y^{\prime}(0)=0 \\
& \text { Taking, } \\
& \qquad y^{\prime \prime}(x)=Y^{T} \Theta(x), \\
& \text { where } \\
& \qquad Y=\left[y_{0,} y_{1,} y_{2,} y_{3,} y_{4,} y_{5,} y_{6,} y_{7,} y_{8,} y_{9,} y_{10}, y_{11}\right]^{T} \\
& \text { and } \\
& \Theta(x)=\left[\phi_{0}, \phi_{1}, \phi_{2}, \psi_{00}^{0}, \psi_{00}^{l}, \psi_{00}^{2}, \psi_{10}^{0}, \psi_{10}^{1}, \psi_{10}^{2}, \psi_{11}^{0}, \psi_{11}^{l}, \psi_{11}^{2}\right]^{T} .
\end{aligned}
$$

From initial condition, we have

$$
\begin{aligned}
y^{\prime}(x) & =Y^{T} P \Theta(x), \quad y(x)=Y^{T} P^{2} \Theta(x) \\
1 & =F^{T} \Theta(x) .
\end{aligned}
$$

and

Using equations (28)-(33) into equation (28) we get

$$
\Theta^{T} Y=\Theta^{T} F
$$

which is satisfy for each $x$ in finite interval, therefore we get

$$
Y=F
$$

and hence

$$
\mathrm{Y}=[1,0,0,0,0,0,0,0,0,0,0,0]^{\mathrm{T}} .
$$

Using equation (33) and we get exact solution

$$
y(x)=\frac{x^{2}}{2} \text {. }
$$

\section{CONCLUSION}

The aim of the present work is to suggest an efficient method for solving non homogeneous differential equation with IVP by reducing an integral equation into a set of algebraic equations with the help of operational matrix. It is also shown the QLMW provides an exact solution. This work shall facilitate in solving real-world problems, such as related to the mathematical physics and digital electronics etc.

\section{REFERENCES}

[1] Daubechies, I., Ten Lectures on Wavelets, SIAM, Philadelphia, PA, 1992.

[2] Ricker, N., The Form and Laws of Propagation of Seismic Wavelets, Geophysics, 18(1953), 10-40.

[3] Robinson, E. A., Random Wavelets and Cybernetic Systems, Griffin and Co., London, 1962.

[4] Robinson, E. A., Wavelet composition of time series, in $\mathrm{H}$. O. A. Wold ed., Econometric model building, essays on the causal chain approach: North Holland Publishing CO., (1964a), 37-106.

[5] Morlet, J., et al, Wave Propagation and Sampling Theory, Part-1, Complex Signal Land Scattering In Multilayer Media, J. Geophys, 47(1982a), 203-221.

[6] Morlet, J., et al, Wave Propagation and Sampling Theory, Part 2: Sampling Theory and Complex Waves, J Geophys, 47(1982b), 222-236.

[7] Debnath, L., Wavelet Transforms and Their Applications, Birkhauser, Boston, 2002.

[8] Resnikoff, H. L. and Wells, R. O. Jr., Wavelet Analysis, Springer-Verlag New York, Inc. 2004.

[9] Babolian, E. and Fattahzdeh, F., Numerical solution of differential equations by using Chebyshev wavelet operational matrix of integration, Applied Mathematics and Computation, 188(2007), 417-426.

[10] Yousefi, S. A., Legendre wavelet method for solving differential equations of Lane- Emden type, Applied Mathematics and Computation, 181(2006), 1417-1422.

[11] Pathak, A., Numerical solution of linear integrodifferential equations by using quadratic Legendre multiwavelet direct method, Advanced Research in Scientific Computing, 4(2012), 1943-2364.

[12] Soman, K. P. and Ramachandran, K. I., Insights Into Wavelets From Theory to Practice, PHI learning, 2010.

[13] Razzaghi, M. and Yousefi, S., The Legendre wavelets operational matrix of integration, International Journal of System Science, 32(2001), 495-502. 